\title{
A Performance Evaluation of Apache Kafka in Support of Big Data Streaming Applications
}

Paul LE NOAC' $H^{1}$, Alexandru COSTAN ${ }^{2}$, Luc BOUGE ${ }^{3}$

\section{Context}

- Stream computing: a new paradigm enabling real-time Big Data processing through 3 steps

- Ingestion: Apache Kafka

- Processing: Apache Spark / Flink

- Storage: HDFS, Cassandra

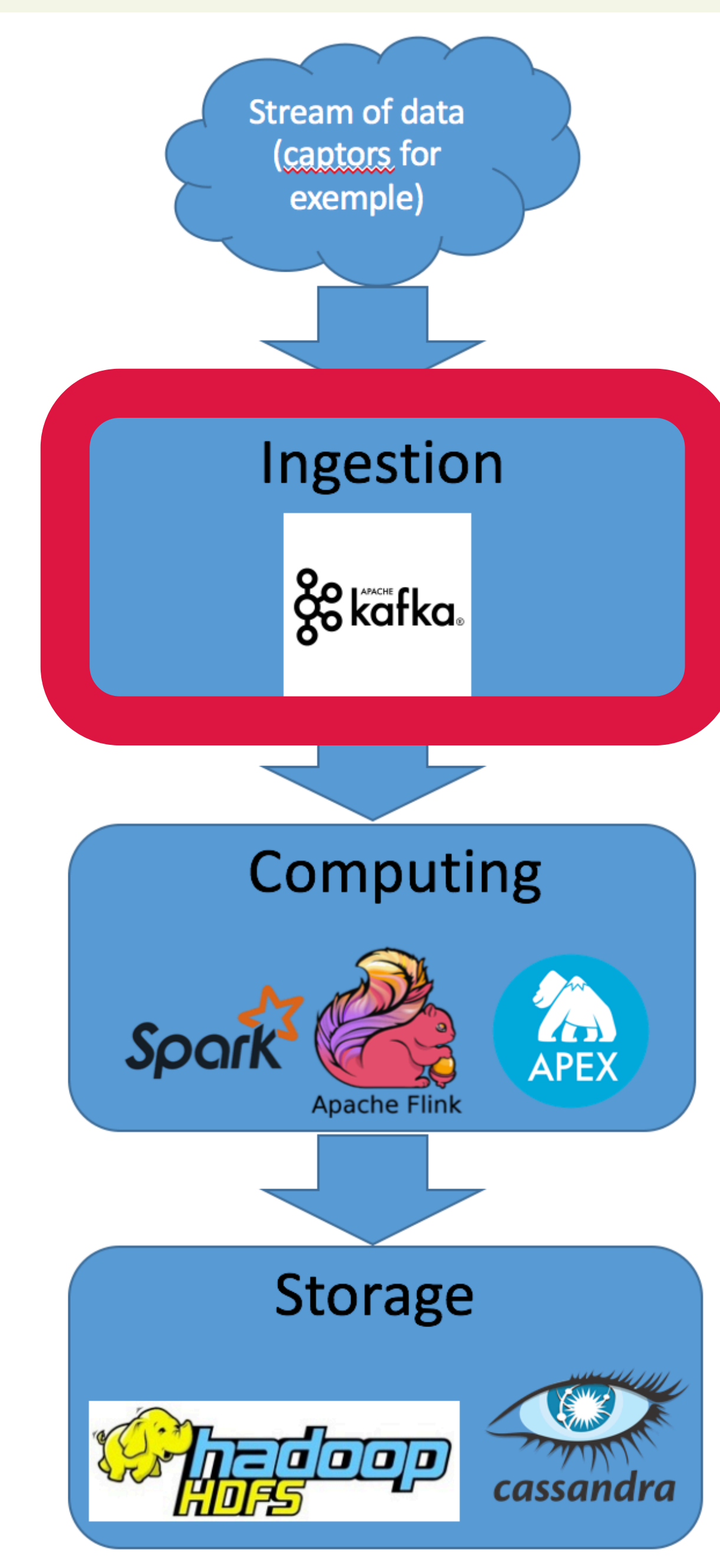

\section{Contribution}

- Identify the impact of different parameter settings on Kafka's overall performance

- Experiment evaluation of several configurations and performance metrics of Kafka

- Allow users to avoid bottlenecks and achieve good practice for stream processing

\section{Results}

Producer performances when modifying batch size for several number of nodes and a message size of $50 B$

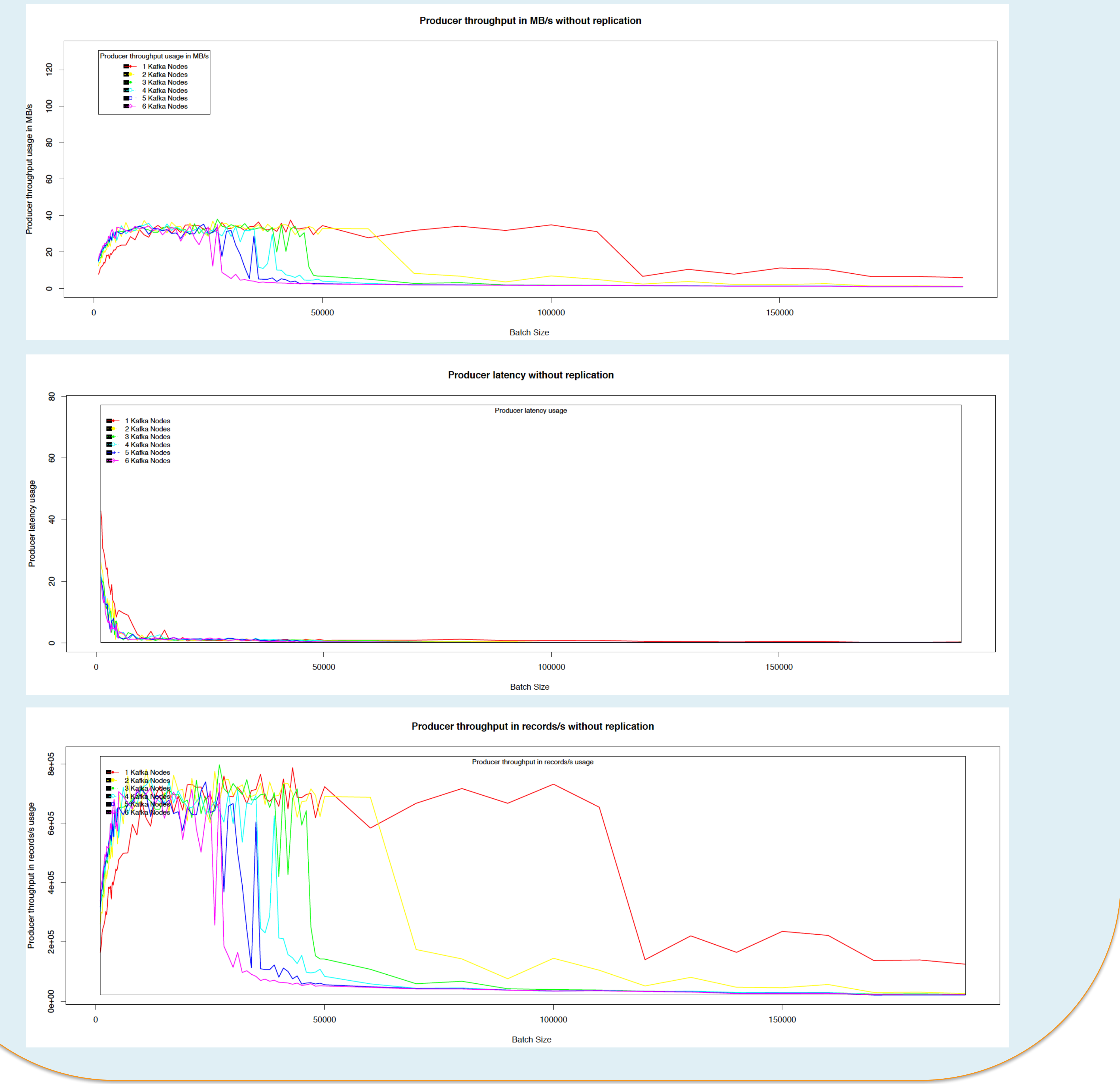

\section{Kafka Architecture}

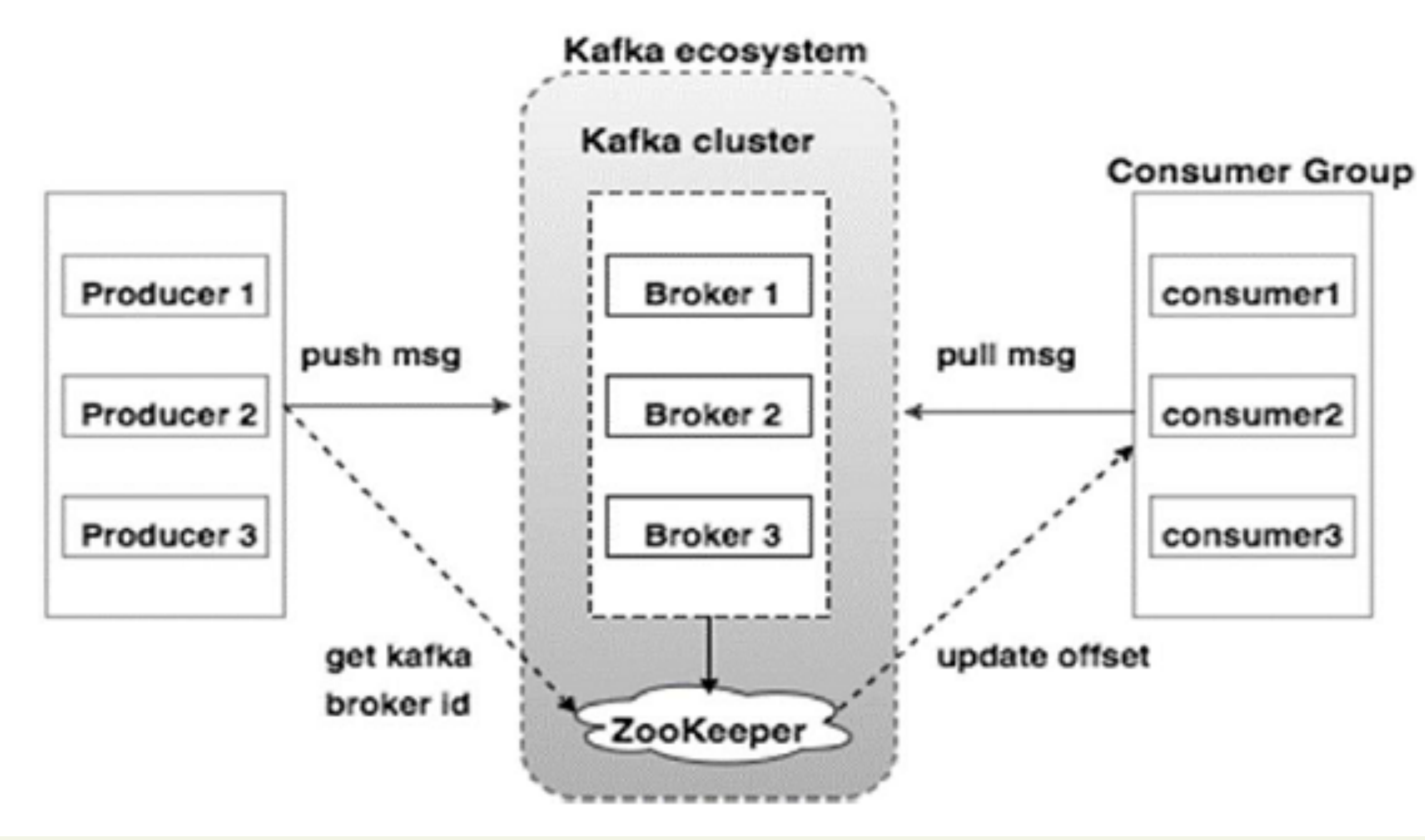

\section{Methodology}

- Isolate the performance of each Kafka component

- Separated tests for Producers and Consumers

- Make correlations between configuration parameters, resource usage and performance metrics

- Experiments executed on Grid5000

- Up to 32 nodes (16 cores per nodes, 28 GB RAM, 10 Gigabit Ethernet)

\section{Key metrics}

\section{Parameters :}

- Message size

- $\quad$ Batch size

- $\quad$ Acquirement strategy

- $\quad$ Network and disk I/O threads

- Message replication

- Hardware

\section{Performance} Metrics:

- Throughput (MB/s, items/s)

- Latency

- CPU usage

- Disk usage

- Memory usage

- Network usage

\section{Take-aways}

- The variation of the batch size shows that there is a range of batches with a better performance.

-When varying the number of nodes in some scenarios: a sudden performance drop (probably due to the internal Kafka synchronizations as well as the underlying network).

- Future work : evaluating reference processing frameworks (Apache Spark and Flink)

Bibliographie / sources

- 1. https://data-artisans.com/blog/extendingthe-yahoo-streaming-benchmark

- 2. http://www.tutorialspoint.com/apache kafka/

Contacts

paul.lenoach@irisa.fr

alexandru.costan@irisa.fr 